\title{
Face-to-Face Meetings with Neurosurgical Patients Before Hospital Discharge: Impact on Telephone Outreach, Emergency Department Visits, and Hospital Readmissions
}

\author{
Franz H. Vergara, PhD, DNP, RN, ONC, CCM, Jean E. Davis, PhD, RN, FAAN, ${ }^{2, *}$ Chakra Budhathoki, PhD, ${ }^{3}$ \\ Nancy J. Sullivan, DNP, RN, ${ }^{3}$ and Daniel J. Sheridan, PhD, RN, FAAN ${ }^{4}$
}

\begin{abstract}
The Johns Hopkins Community Health Partnerships (JCHiP) was developed in 2010 within the Johns Hopkins Health Systems. As part of JCHiP, the Patient Access Line call center was created. The average telephone reach rate at The Johns Hopkins Hospital in 2014 was only 53\%. In a population of adult neurosurgical patients, this study aimed to: determine the impact of face-to-face meetings with neurosurgical patients before hospital discharge on telephone follow-up (TFU) reach rates, and determine the association between TFU reach rates and subsequent emergency department (ED) visits and hospital readmission rates. This quasi-experimental study used a posttestonly research design with a comparison group. Two adult inpatient neurosurgical units at the Johns Hopkins Hospital were selected as the intervention and comparison groups. A convenience sampling technique was used. Face-to-face meetings pre hospital discharge resulted in a TFU reach rate of $97.7 \%$ on the intervention unit while the comparison unit had only a 76.1\% TFU reach rate $(P<.001)$. Reached patients had fewer ED visits $(7.8 \%)$ than not reached patients $(17.4 \%)$; however, the difference was not statistically significant $(P=.138)$. Reached patients also had fewer hospital readmissions $(3.3 \%)$ than not reached patients $(8.7 \%)$; this also was not statistically significant $(P=.214)$. This study demonstrated that face-to-face meetings with neurosurgical patients prior to discharge increased TFU rates. Results were statistically significant. ED visits and hospital readmissions were also reduced in reached patients and the findings were clinically significant.
\end{abstract}

Keywords: telephone follow-up, reach rate, readmissions, emergency visits, face-to-face meetings

\section{Introduction}

I N 2010, THE Centers for Medicare \& Medicaid Services awarded the Johns Hopkins Health Systems a grant to create and design a comprehensive and integrated health care program, the Johns Hopkins Community Health Partnerships (JCHiP). JCHiP was a complementary bundle of interventions with primary and secondary drivers (Figure 1) designed to improve access to care for high-risk adults and reduce hospital readmissions in East Baltimore, MD. ${ }^{1}$ When deployed, it was associated with lower spending and improved health care outcomes by reducing an aggregate total cost of care of \$59.8 million for Medicare and Medicaid participants. $^{2}$

As a part of JCHiP, the Patient Access Line (PAL) call center was created to serve as a secondary driver to promote seamless transitions of care from hospital to home. ${ }^{3}$ Hsiao et $\mathrm{al}^{4}$ published a detailed explanation of the implementation of JCHiP and the PAL call center in a recent study. Initially, the PAL call center was staffed by 5 telephonic

${ }^{1}$ Bloomberg School of Public Health, The Johns Hopkins University, Baltimore, Maryland.

${ }^{2}$ Goldfarb School of Nursing at Barnes-Jewish College, St. Louis, Missouri.

${ }^{3}$ School of Nursing, The Johns Hopkins University, Baltimore, Maryland.

${ }^{4}$ Texas A\&M University, College of Nursing, Bryan, Texas.

*Current affiliation: College of Nursing, University of South Carolina, Columbia, South Carolina.

(C) Franz H. Vergara et al. 2019; Published by Mary Ann Liebert, Inc. This Open Access article is distributed under the terms of the Creative Commons License (http://creativecommons.org/licenses/by/4.0), which permits unrestricted use, distribution, and reproduction in any medium, provided the original work is properly cited. 


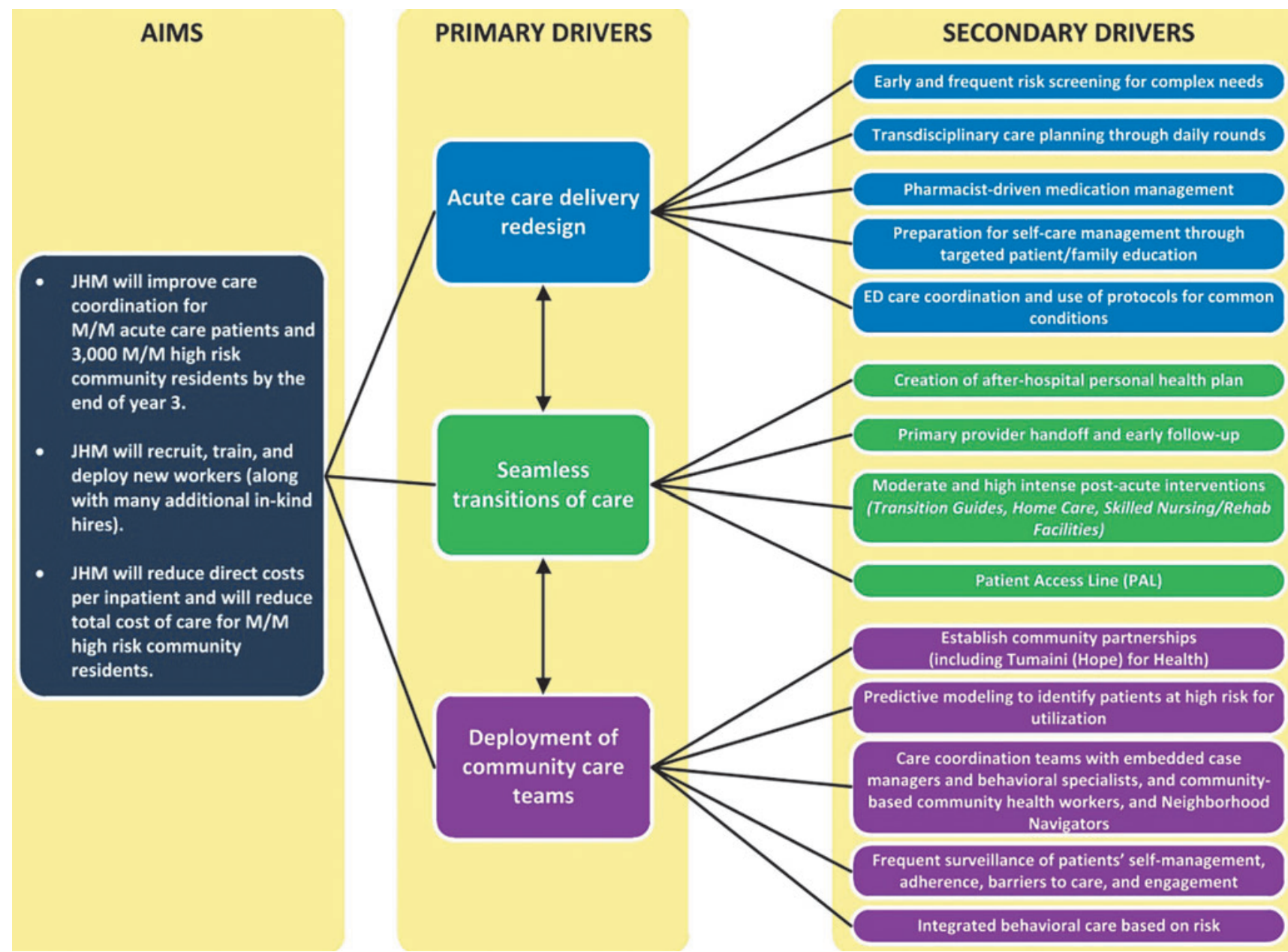

FIG. 1. JCHiP driver diagram for care coordination. ED, emergency department; JHM, Johns Hopkins Medicine; M/M, Medicare/Medicaid.

registered nurse case managers with the primary goal of conducting telephone follow-up (TFU) post hospital discharge. The average TFU reach rate at Johns Hopkins Hospital in 2014 was 53\%. ${ }^{5}$ There were very few published studies on improving telephone outreach. Most studies focused on medical patients. ${ }^{6,7}$ Rates of telephone outreach were unknown for neurosurgical patients. Also, there were few recent studies linking TFU to subsequent emergency department (ED) visits and 30-day hospital readmissions. ${ }^{3,4,8}$ Hoyer et al emphasized that "patients at highest risk for rehospitalization were also the least likely to receive the assigned care coordination intervention, ", (p 626) such as the PAL call. Therefore, it is imperative to develop methods to engage the hardest to reach patients to fully deploy the various drivers of the JCHiP program such as TFU.

The aims of this study were to:

1. Determine the impact of a pre hospital discharge faceto-face meeting on the post hospital TFU telephone reach rate of neurosurgical patients; and

2. Determine the association of successfully reaching patients post hospital discharge with subsequent ED visits and readmission rates of patients admitted to an adult neurosurgical patients unit.

\section{Methods}

\section{Study design and setting}

A prospective posttest-only quasi-experimental research design with a comparison group was used to address the study aims. To examine causality, a quasi-experimental design was selected, as true randomization was not feasible during the study time period and randomly allocating participants to intervention and comparison groups was not practical. ${ }^{9}$ A convenience sampling technique was employed as a measure to recruit as many participants as possible to increase the power of the study.

The study was conducted on 2 neurosurgical units (named Unit A and Unit B for study purposes) servicing similar populations at the Johns Hopkins Hospital. After flipping a coin, Unit A was selected as the intervention ward, with Unit B serving as the comparison.

\section{Intervention}

Face-to-face meeting intervention. The study used a 1time, face-to-face meeting intervention conducted by the corresponding author that lasted approximately 6-10 minutes for each participant. Face-to face meeting as an intervention was guided by the elements of the Transitions Theory. ${ }^{10}$ At each face-to-face meeting intervention the investigator: 
- introduced himself and greeted the patient by shaking hands;

- asked the patient's permission to sit down so that the investigator could speak without looking down at the patient;

- informed the patient of the purpose of TFU post hospital discharge; and

- assisted the patient with completing a patient handout that would be used by the investigator to contact the patient post hospital discharge.

The patient handout requested the following information:

- the best phone number(s) to reach the patient;

- the best time and date for TFU; and

- a reminder of paperwork and items needed at the time of the phone call.

Each patient signed the patient handout and took this document with her/him upon discharge. This both demonstrated agreement about the scheduled TFU and served as an appointment card for the TFU. The photocopy of the signed patient handout was de-identified, and a unique identification code was assigned.

Twenty-four to 72 hours after hospital discharge, a PAL call was conducted for all eligible patients based on the date and time agreed to during the face-to-face meeting. If participants were not reached at the agreed-upon time for the telephone call, 2 more attempts were made to reach the patient telephonically. After 3 telephone call attempts, if the patient did not answer or was unavailable, attempts to conduct TFU were discontinued.

Routine care. Patients admitted to the comparison group received care as usual, which included up to 3 TFU call attempts made by telephonic nurse case managers without meeting patients prior to hospital discharge. The traditional workflow process and task list for routine care included the following:

- Creating daily unit-based discharge reports from Research Electronic Data Capture (REDCap);

- Conducting post hospital discharge telephone calls using a prescribed script;

- Contacting the discharging medical providers, home care services, or pharmacists as indicated;

- Answering questions about self-care management needs; and

- Documenting the TFU in the electronic medical record (EMR) at the end of the telephone call.

Intervention and comparison groups both received the routine care of TFU post hospital discharge. However, the comparison group did not receive a face-to-face meeting prior to hospital discharge and TFU.

\section{Study sample, screening, and enrollment}

The intervention and comparison units had a similar mix of patients and used a novel care coordination program from JCHiP, with TFU being a component of discharge planning. Eligible patients in this research study met the following criteria:

- postoperative patients on the neurosurgical wards;

- low-to-moderate risk of hospital readmission;

- ages 18 years or older; and

- speak and understand English.
Patients with the following post hospital discharge needs and conditions were excluded:

- need for skilled nursing home care or transition guide services;

- planned transfer to a skilled nursing facility, rehabilitation unit, or assisted living facility;

- readmitted patients from another nursing unit or hospital;

- case management by Johns Hopkins International (JHI) staff;

- prior referral to hospice or palliative care agencies;

- left hospital against medical advice; and

- lacking capacity to consent.

Enrollment. Face-to-face meeting interventions were conducted at the intervention unit from June 13, 2016, to September 16, 2016. The number of face-to-face meeting interventions per week varied depending on the number of eligible patients for each day of data collection. The Consolidated Standards of Reporting Trials (CONSORT) flow diagram of Kuriyama et $\mathrm{al}^{11}$ was used to elucidate the screening strategies for eligible participants (Figure 2). A total of 758 patients were screened as possible research participants. After using the random selection functionality of IBM SPSS (IBM Corporation, Armonk, NY) ${ }^{12}$ the intervention and comparison groups were each allocated 88 subjects.

While conducting face-to-face meetings with the intervention group, a retrospective chart review was conducted to determine the independence of research participants because of the probability of hospital readmission of patients in the comparison group. The review prevented enrolling research participants twice between the intervention and comparison groups. Therefore, the subjects in each group remained mutually exclusive.

\section{Statistical analysis}

A power analysis was conducted a priori to ensure sufficient sample size to achieve adequate power. ${ }^{9}$ Sample size estimates using medium-range effect sizes were calculated using the Power Analysis and Sample Size online computer software based on Cohen's ${ }^{13}$ formula. A medium effect size of 0.30 using a 1 degree-of-freedom chi-square test with a significance level of 0.05 was selected to obtain a power of $80 \%$. Based on the estimated medium effect size as calculated, at least 88 participants were needed in each group (intervention and comparison units), for a total minimum of 176 patients. Nonparametric statistical methods (Pearson chi-square test and Fisher exact tests) were employed to determine any differences between categorical sociodemographic and surgical variables. Nominal data were analyzed as to whether the patient was reached or not reached (yes or no). Pearson chi-square test and cross tabulations were used to compare proportions of reached or not reached patients, patients who had an ED visit or not, and patients readmitted or not. ${ }^{14}$

\section{Data collection}

Data were collected and managed using REDCap, a secure, web-based software application for collecting research information. ${ }^{15}$ REDCap is the internal documentation system of the PAL department. In addition, a retrospective EMR review (using EPIC; Epic Systems Corporation, Verona, WI) was conducted 30 days after hospital discharge for each research participant from the intervention and comparison units. The EMR review determined: 


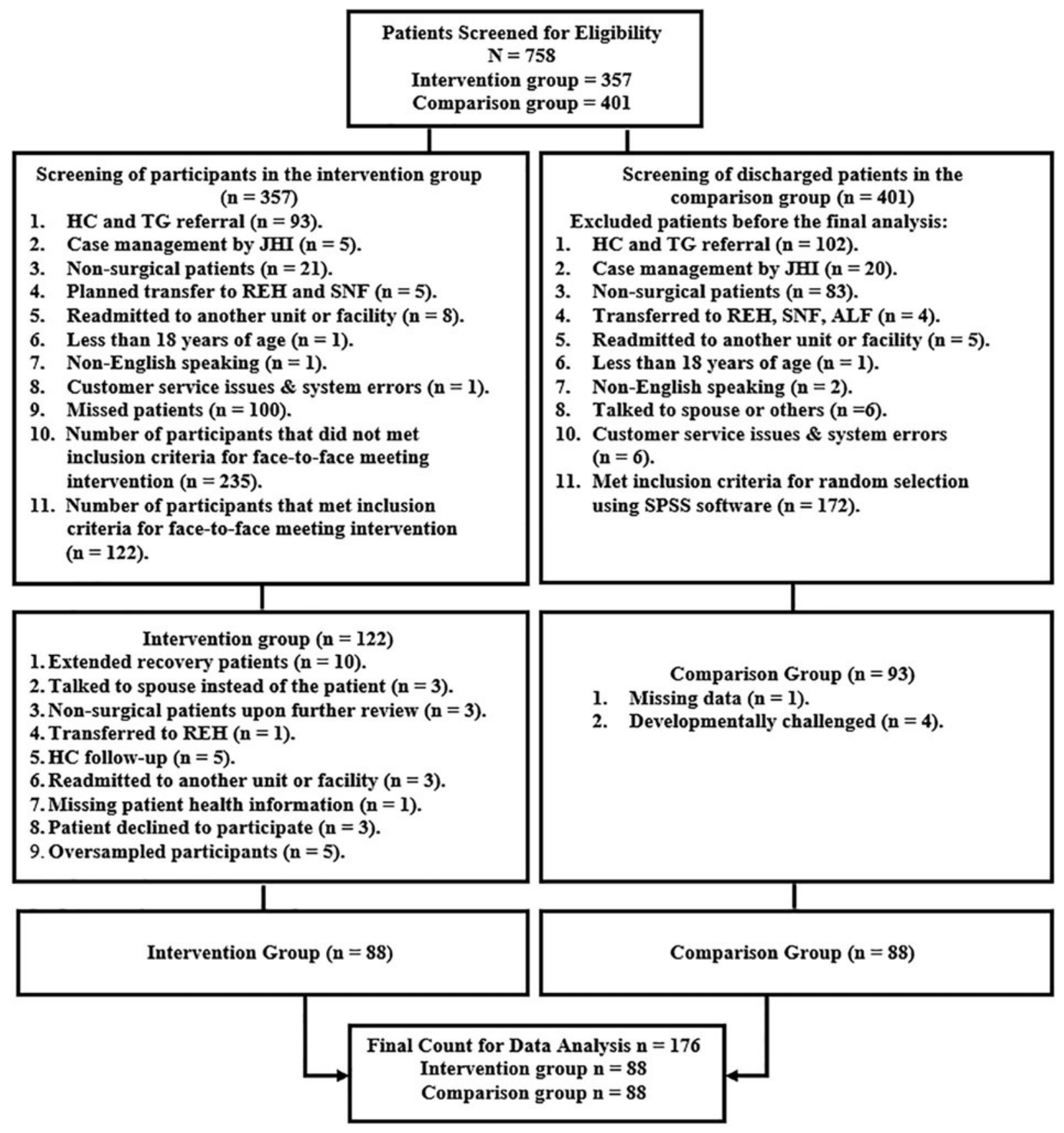

FIG. 2. CONSORT flow diagram for screening eligible patients. ALF, assisted living facility; HC, home care; JHI, Johns Hopkins International; REH, rehabilitation unit; SNF, skilled nursing facility; TG, transition guide.

- how many patients were either reached or not reached by TFU;

- how many phone call attempts were made to reach the patients;

- how many and which patients visited or did not visit the ED within 30 days of hospital discharge; and

- how many and which patients were readmitted or not readmitted 30 days after hospital discharge.

\section{Ethical consideration and data security}

An Institutional Review Board application was submitted to Johns Hopkins Medicine and this study was classified as a quality improvement and deemed exempt for full review because of its less than minimal risk to participants. Data were de-identified through substitution by creating a reidentification table. ${ }^{16}$ The folders containing the reidentification table and master keys table were saved on the firewalled and protected server of the Johns Hopkins Medical Institutions.

\section{Results}

Of the 15 sociodemographic and background variables, 4 demonstrated a significant difference between the intervention and comparison groups. Employment status $(P=.014)$, educational attainment $(P=.002)$, having children younger than age 18 years living at home $(P=.010)$, and hospital 
service $(P=.039)$ were significantly different between the intervention and comparison groups (Table 1).

The intervention group, who received face-to-face meeting interventions, had a TFU reach rate that was $21.6 \%$ higher than that of the comparison group. This indicated that pre hospital discharge face-to-face meeting interventions increased TFU reach rates, and Pearson's chi-square test demonstrated very high statistical significance $(P=.000)$ (Table 2$)$.

The researchers also conducted a statistical analysis comparing the number of TFU attempts to successfully reach the patient. Close to $30 \%$ more patients on the intervention unit were reached during the first attempt compared to the comparison unit, which demonstrated statistical significance $(P=.001)$ (Table 2$)$. Interestingly, $4-5$ call attempts were made to some patients $(n=5 ; 7.5 \%)$ on the comparison unit by other case managers trying to reach the patients successfully but this low number did not affect the results of this analysis (Table 2).

Table 3 describes the cross tabulation of subsequent ED visits of reached and not reached participants from the intervention and comparison groups. Participants in the intervention group who were reached successfully had $4.6 \%$ fewer subsequent ED visits than those in the comparison group who were successfully reached. The total ED visits of the reached participants were $7.8 \%(n=12)$.

However, the ED visit rate for participants in the intervention group who were not successfully reached was close to $36 \%$ higher than that of the comparison group who were not reached. The total percentage of ED visits for participants not reached is $17.4 \%(n=4)$, which is close to $10 \%$ higher in comparison to those patients who were successfully reached (Table 3 ).

Pearson's chi-square test also was also employed to determine whether successfully reaching adult surgical patients post hospital discharge would decrease the number of subsequent 30-day ED visits. The percentage of patients who were reached had lower rates of ED visits compared to those who were not reached, but the difference was not statistically significant (reached participants, $P=.290$; not reached participants, $P=.203$ ). Total ED visits also were analyzed and did not show a statistically significant difference between the groups $(P=.294)$ (Table 3$)$.

Because some cells contained 5 to 9 expected cases or counts and some cells had fewer than 5 expected count or cases, a Fisher exact test and Yates' continuity correction also were employed as a measure to ensure accuracy of results. For reached participants, Fisher exact test $(P=.368)$ and Yates' continuity correction $(P=.450)$ did not demonstrate any statistical significance. For not reached participants, Fisher exact test $(P=.324)$ and Yates' continuity correction $(P=.766)$ did not demonstrate any statistical significance. In addition, statistical significance was not demonstrated in total ED visits between the intervention and comparison group after employing a Fisher exact test $(P=.292)$ and Yates' continuity correction $(P=.432)$. Although not statistically significant, this finding is clinically significant and needs further investigation and research (Table 3 ).

\section{Discussion}

This study is the first to examine TFU among neurosurgical patients. This also is the first study to employ inferential statistics to determine the effect of face-to-face meetings on telephone outreach for neurosurgical patients. The TFU reach rate for the intervention group was significantly greater $(97.7 \%)$ than the reach rate for the comparison group $(76.1 \%)$, with a difference of $21.6 \%$ and a very high statistical significance $(P=.001)$. The $97.7 \%$ TFU reach rate was similar to that of a previous study that utilized face-to-face meetings before hospital discharge for medical patients. ${ }^{6}$ Past studies demonstrated TFU reach rates between $86 \%$ and $99 \%$ in medical patients. ${ }^{6,17-20}$ When compared to the average TFU reach rates $(58 \%)$ of medical patients who did not receive face-to-face meeting interventions at the Johns Hopkins Hospital, ${ }^{6}$ the baseline reach rate for neurosurgical patients was still very high $(76.1 \%)$ even though face-to-face meetings were not employed. Furthermore, the $76.1 \%$ TFU reach rate for the comparison group in this study was higher than the national average TFU reach rate $(40 \%)$ in the United States $^{21}$ and higher than the average TFU reach rate $(68 \%)$ of a comparison group in a study conducted in an ED setting. ${ }^{22}$ This finding that, even without face-to-face meetings, TFU reach rates of neurosurgical patients were higher compared to TFU reach rates of medical and ED patients is clinically significant. The reasons for the underlying baseline difference of TFU reach rates between medical, ED, and neurosurgical patients are unknown, and warrant further research. Given that this is the first study of neurosurgical patients, replication of the study in a multisite setting and with different clinical specialties is highly recommended. Also, further investigation is needed to determine if sociodemographic variables and chronicity of illness between medical and neurosurgical patients are factors that affect patients being reached or not reached.

This study also explained that conducting pre hospital discharge face-to-face meetings may reduce the number of TFU attempts to successfully reach patients post hospital discharge. Many patients in the intervention group $(n=63$; $71.6 \%$ ) were reached during the first phone call attempt compared to the comparison group $(n=37 ; 42 \%)$, which demonstrated high statistical significance $(P=.001)$. Most importantly, this is the first study to demonstrate that faceto-face meetings may reduce case managers' efforts to reach patients after hospital discharge.

The study findings demonstrated clinical significance but not statistical significance when examining ED visits and hospital readmissions rates, but the outcomes trended toward significance and are consistent with recent larger studies within the organization. ${ }^{3,4,8}$ These findings also were comparable to national benchmark clinical outcomes. Nationally, several studies and systematic reviews found that in the United States, readmission of neurosurgical patients with cranial procedures was between $6.9 \%$ and $23.89 \%$. $^{23-26}$ Average 30-day readmission rates following a neurosurgical spine procedure were between $4.2 \%$ and $7.4 \% .^{27}$

Readmissions of orthopedic spinal surgery patients varied by procedure but were generally lower than readmission rates of neurosurgical patients with cranial procedures and neurosurgical spine procedures. For example, readmission rates of patients who had orthopedic lumbar discectomies were $2.6 \%,{ }^{28}$ and rates of patients who had lumbar fusions were around $2.9 \% .^{29}$ Nevertheless, the overall risks for neurosurgical and orthopedic complications and all-cause readmissions were similar across all types of procedures in a recently conducted retrospective longitudinal study. ${ }^{30}$ 
Table 1. Sociodemographic Variables of Participants and Differences Between Groups $(\mathrm{N}=176)$

\begin{tabular}{|c|c|c|c|c|c|c|c|}
\hline \multirow[b]{2}{*}{ Characteristics } & \multicolumn{2}{|c|}{ Intervention $(n=88)$} & \multicolumn{2}{|c|}{ Comparison $(n=88)$} & \multicolumn{2}{|c|}{ Total } & \multirow[b]{2}{*}{$\mathrm{P}$} \\
\hline & $n$ & $\%$ & $n$ & $\%$ & $N$ & $\%$ & \\
\hline Age in Years & & & & & & & $0.939^{\mathrm{a}}$ \\
\hline $18-29$ & 9 & 10.2 & 12 & 13.6 & 21 & 11.9 & \\
\hline $30-39$ & 13 & 14.8 & 11 & 12.5 & 24 & 13.6 & \\
\hline $40-49$ & 22 & 25.0 & 17 & 19.3 & 39 & 22.2 & \\
\hline $50-59$ & 20 & 22.7 & 23 & 26.1 & 43 & 24.4 & \\
\hline $60-69$ & 17 & 19.3 & 16 & 18.2 & 33 & 18.8 & \\
\hline $70-79$ & 5 & 5.7 & 7 & 8.0 & 12 & 6.8 & \\
\hline$\geq 80$ & 2 & 2.3 & 2 & 2.3 & 4 & 2.3 & \\
\hline Sex & & & & & & & $1.000^{\mathrm{b}}$ \\
\hline Male & 38 & 43.2 & 38 & 43.2 & 76 & 43.2 & \\
\hline Female & 50 & 56.8 & 50 & 56.8 & 100 & 56.8 & \\
\hline Race & & & & & & & $0.833^{\mathrm{a}}$ \\
\hline African American & 9 & 10.2 & 12 & 13.6 & 21 & 11.9 & \\
\hline White & 73 & 83.0 & 71 & 80.7 & 144 & 81.8 & \\
\hline Others & 6 & 6.8 & 5 & 5.7 & 11 & 6.3 & \\
\hline Educational Attainment & & & & & & & $0.002^{\mathrm{a}^{*}}$ \\
\hline Less than high school & 1 & 1.1 & 1 & 1.1 & 2 & 1.1 & \\
\hline Some high school & 1 & 1.1 & 1 & 1.1 & 2 & 1.1 & \\
\hline High school graduate & 13 & 14.8 & 9 & 10.2 & 22 & 12.5 & \\
\hline Some college & 9 & 10.2 & 14 & 15.9 & 23 & 13.1 & \\
\hline Four-year college graduate or higher & 51 & 58.0 & 30 & 34.1 & 81 & 46.0 & \\
\hline No answer & 13 & 14.8 & 33 & 37.5 & 46 & 26.1 & \\
\hline Employment Status & & & & & & & $0.014^{\mathrm{a}^{*}}$ \\
\hline Employed & 62 & 70.5 & 42 & 47.7 & 104 & 59.1 & \\
\hline Retired & 10 & 11.4 & 13 & 14.8 & 23 & 13.1 & \\
\hline Disabled & 1 & 1.1 & 2 & 2.3 & 3 & 1.7 & \\
\hline Unemployed & 15 & 17.0 & 29 & 33.0 & 44 & 25.0 & \\
\hline No answer or unknown & 0 & 0.0 & 2 & 2.3 & 2 & 1.1 & \\
\hline Marital Status & & & & & & & $0.151^{\mathrm{a}}$ \\
\hline Single & 15 & 17.0 & 19 & 21.6 & 34 & 19.3 & \\
\hline Married & 66 & 75.0 & 60 & 68.2 & 126 & 71.6 & \\
\hline Widowed & 1 & 1.1 & 6 & 6.8 & 7 & 4.0 & \\
\hline Divorced/Separated & 6 & 6.8 & 3 & 3.4 & 9 & 5.1 & \\
\hline Children $<18$ years old & & & & & & & $0.010^{b *}$ \\
\hline No & 57 & 64.8 & 73 & 83.0 & 130 & 73.9 & \\
\hline Yes & 31 & 35.2 & 15 & 17.0 & 46 & 26.1 & \\
\hline Primary Insurance Status & & & & & & & $0.215^{\mathrm{b}}$ \\
\hline Public & 17 & 19.3 & 25 & 28.4 & 42 & 23.9 & \\
\hline Private & 71 & 80.7 & 63 & 71.6 & 134 & 76.1 & \\
\hline Housing Status & & & & & & & $0.307^{\mathrm{b}}$ \\
\hline Lives alone & 6 & 6.8 & 11 & 12.5 & 17 & 9.7 & \\
\hline Lives with family or significant other & 82 & 93.2 & 77 & 87.5 & 159 & 90.3 & \\
\hline Religious Affiliation & & & & & & & $0.722^{\mathrm{a}}$ \\
\hline Christianity & 41 & 46.6 & 47 & 53.4 & 88 & 50.0 & \\
\hline Jewish & 7 & 8.0 & 4 & 4.5 & 11 & 6.3 & \\
\hline Other (No answer or unknown) & 15 & 17.0 & 13 & 14.8 & 28 & 15.9 & \\
\hline None & 25 & 28.4 & 24 & 27.3 & 49 & 27.8 & \\
\hline Admission Type & & & & & & & $.331^{\mathrm{b}}$ \\
\hline Emergency & 7 & 8.0 & 12 & 13.6 & 19 & 10.8 & \\
\hline Elective & 81 & 92.0 & 76 & 86.4 & 157 & 189.2 & \\
\hline Hospital Service & & & & & & & $.039^{\mathrm{a}^{*}}$ \\
\hline Neurosurgery, brain tumor & 45 & 51.1 & 44 & 50.0 & 89 & 50.6 & \\
\hline Neurosurgery, spine & 26 & 29.5 & 15 & 17.0 & 41 & 23.3 & \\
\hline Neurosurgery, vascular & 12 & 13.6 & 15 & 17.0 & 27 & 15.3 & \\
\hline Orthopedic Surgery, spine & 4 & 4.5 & 5 & 5.7 & 9 & 5.1 & \\
\hline Others & 1 & 1.1 & 9 & 10.2 & 10 & 5.7 & \\
\hline
\end{tabular}


TABle 1. (CONTINUED)

\begin{tabular}{|c|c|c|c|c|c|c|c|}
\hline \multirow[b]{2}{*}{ Characteristics } & \multicolumn{2}{|c|}{ Intervention $(n=88)$} & \multicolumn{2}{|c|}{ Comparison $(n=88)$} & \multicolumn{2}{|c|}{ Total } & \multirow[b]{2}{*}{$\mathrm{P}$} \\
\hline & $n$ & $\%$ & $n$ & $\%$ & $N$ & $\%$ & \\
\hline Surgical Procedures & & & & & & & $.060^{\mathrm{a}}$ \\
\hline Craniectomy & 5 & 5.7 & 11 & 12.5 & 16 & 9.1 & \\
\hline Craniotomy & 28 & 31.8 & 24 & 27.3 & 52 & 29.5 & \\
\hline Microvascular decompression & 7 & 8.0 & 2 & 2.3 & 9 & 5.1 & \\
\hline Decompression and fusion & 3 & 3.4 & 3 & 3.4 & 6 & 3.4 & \\
\hline Deep brain stimulator placement & 2 & 2.3 & 0 & 0.0 & 2 & 1.1 & \\
\hline Endoscopic resection of tumor & 8 & 9.1 & 10 & 11.4 & 18 & 10.2 & \\
\hline Laminectomies, discectomies, and fusions & 24 & 27.3 & 16 & 18.2 & 40 & 22.7 & \\
\hline Placement of epidural blood patch & 2 & 2.3 & 1 & 1.1 & 3 & 1.7 & \\
\hline Ventriculoperitoneal shunt and revision & 3 & 3.4 & 6 & 6.8 & 9 & 5.1 & \\
\hline Other surgical procedures & 6 & 6.8 & 9 & 10.2 & 15 & 8.5 & \\
\hline Cranioplasty & 0 & 0.0 & 6 & 6.8 & 6 & 3.4 & \\
\hline ESDP scores $\geq 10$ & & & & & & & $.827^{\mathrm{b}}$ \\
\hline No & 75 & 85.2 & 77 & 87.5 & 152 & 86.4 & \\
\hline Yes & 13 & 14.8 & 11 & 12.5 & 24 & 13.6 & \\
\hline Length of stay & & & & & & & $.809^{a}$ \\
\hline 1-7 days & 81 & 92 & 82 & 93.2 & 163 & 92.6 & \\
\hline 8-14 days & 6 & 6.8 & 4 & 4.5 & 10 & 5.7 & \\
\hline$\geq 15$ days & 1 & 1.1 & 2 & 2.3 & 3 & 1.7 & \\
\hline
\end{tabular}

${ }^{\text {a}}$ Fisher exact test; ${ }^{\text {b}}$ Pearson's chi-square test; statistical significance $* P<.05$. $P$ values in bold are statistically significant or significant. ESDP, early screening for discharge planning.

It also is important to understand the financial implication of TFU because of the current reimbursement trends and push for value-based care. Although the present study did not aim to calculate the specific financial impact of TFU on the neurosurgical ward, several studies within the researchers' organization demonstrated the value of TFU post hospital discharge as a part of a care coordination program. ${ }^{2,3,4,6,8}$ Overall, combining acute care and community interventions, the JCHiP program was associated with $\$ 113.3$ million in cost savings between 2012-2016. ${ }^{2}$ Specifically, for the TFU service of the PAL department, 41,700 patients were served since 2013, covering 3 hospitals. There was a $29 \%$ relative reduction in readmissions from 2014 2017, which is equivalent to 777 readmissions prevented, totaling \$11.8 million in cost savings. ${ }^{2,31}$ These cost reductions were achieved with 9 full-time PAL case managers who reached approximately $53 \%$ of eligible patients for TFU. ${ }^{5}$ There is room for improvement and huge potential for the TFU service to reduce costs exponentially if more patients are contacted, and patients who are hardest to reach and most likely to be readmitted are successfully reached.

\section{Limitations}

The main limitation of this study was the lack of true randomization. This quasi-experimental study utilized a convenience sampling technique to obtain intervention group participants for face-to-face meetings. The design was unable to determine all preexisting factors that might have influenced the outcome of the results. For example, Early Screening for Discharge Planning Scores ${ }^{32}$ (whereby higher scores indicate potential need for special discharge

Table 2. Telephone Follow-Up Reach Rates and Phone Call Attempts

\begin{tabular}{|c|c|c|c|c|c|c|c|}
\hline \multirow[b]{2}{*}{ Call status } & \multicolumn{2}{|c|}{ Intervention $(n=88)$} & \multicolumn{2}{|c|}{ Comparison $(n=88)$} & \multicolumn{2}{|c|}{ Total } & \multirow[b]{2}{*}{$\mathrm{P}$} \\
\hline & $n$ & $\%$ & $n$ & $\%$ & $N$ & $\%$ & \\
\hline Reach rate & & & & & & & $.000^{\mathrm{a}}$ \\
\hline Not reached & 2 & 2.3 & 21 & 23.9 & 23 & 13.1 & \\
\hline Reached & 86 & 97.7 & 67 & 76.1 & 153 & 86.9 & \\
\hline Total & 88 & 100 & 88 & 100.0 & 176 & 100.0 & \\
\hline Number of phone call attempts & & & & & & & $.001^{\mathrm{a}}$ \\
\hline One & 63 & 71.6 & 37 & 42 & 100 & 56.8 & \\
\hline Two & 15 & 17 & 27 & 30.7 & 42 & 23.9 & \\
\hline Three & 10 & 11.4 & 19 & 21.6 & 29 & 16.5 & \\
\hline Four & 0 & 0 & 3 & 3.4 & 3 & 1.7 & \\
\hline Five & 0 & 0 & 2 & 2.3 & 2 & 1.1 & \\
\hline Total & 88 & 100 & 88 & 100.0 & 176 & 100.0 & \\
\hline
\end{tabular}

\footnotetext{
${ }^{\text {a}}$ Pearson's chi-square test. $P$ values in bold are statistically significant.
} 
Table 3. Telephone Follow-Up Reach Rates, Emergency Department Visits, and READMisSion Rates BetweEn Groups

\begin{tabular}{|c|c|c|c|c|c|c|c|}
\hline \multirow{2}{*}{ Call status } & \multicolumn{2}{|c|}{ Intervention $(n=88)$} & \multicolumn{2}{|c|}{ Comparison $(n=88)$} & \multicolumn{2}{|c|}{ Total } & \multirow[b]{2}{*}{$\mathrm{P}$} \\
\hline & $n$ & $\%$ & $n$ & $\%$ & $N$ & $\%$ & \\
\hline Reached & & & & & & & $.290^{\mathrm{b}} ; .368^{\mathrm{a}} ; .450^{\mathrm{c}}$ \\
\hline With ED visit & 5 & 5.8 & 7 & 10.4 & 12 & 7.8 & \\
\hline Without ED visit & 81 & 94.2 & 60 & 89.6 & 141 & 92.2 & \\
\hline Not reached & & & & & & & $.203^{\mathrm{b}} ; .324^{\mathrm{a}} ; .766^{\mathrm{c}}$ \\
\hline With ED visit & 1 & 50.0 & 3 & 14.3 & 4 & 17.4 & \\
\hline Without ED visit & 1 & 50.0 & 18 & 85.7 & 19 & 82.6 & \\
\hline Total ED visits & & & & & & & $.294^{\mathrm{b}} ; .292^{\mathrm{a}} ; .432^{\mathrm{c}}$ \\
\hline With ED visit & 6 & 6.8 & 10 & 11.4 & 16 & 9.1 & \\
\hline Without ED visit & 82 & 93.2 & 78 & 88.6 & 160 & 90.9 & \\
\hline Reached & & & & & & & $.276^{\mathrm{b}} ; .255^{\mathrm{a}} ; .527^{\mathrm{c}}$ \\
\hline Readmitted & 4 & 4.7 & 1 & 1.5 & 5 & 3.3 & \\
\hline Not readmitted & 82 & 95.3 & 66 & 98.5 & 148 & 96.7 & \\
\hline Not reached & & & & & & & $.648^{\mathrm{b}} ; 1.000^{\mathrm{a}} ; 1.000^{\mathrm{c}}$ \\
\hline Readmitted & 0 & 0.0 & 2 & 9.5 & 2 & 8.7 & \\
\hline Not readmitted & 2 & 100.0 & 19 & 90.5 & 21 & 91.3 & \\
\hline Total Readmissions & & & & & & & $.700^{\mathrm{b}} ; .699^{\mathrm{a}} ; 1.000^{\mathrm{c}}$ \\
\hline Readmitted & 4 & 4.5 & 3 & 3.4 & 7 & 4.0 & \\
\hline Not readmitted & 84 & 95.5 & 85 & 96.6 & 169 & 96.0 & \\
\hline
\end{tabular}

${ }^{\mathrm{a}}$ Fisher exact test; ${ }^{\mathrm{b}}$ Pearson's chi-square; ' Yates' continuity correction of the chi-square.

services) were not considered before enrollment in the study because all patients, even those who declined the recommended high-intensity post-discharge services (eg, home care nurse visit, transition guide services) still received TFU from the telephonic nurse case manager.

Also, it was challenging to control the number of phone call attempts of other case managers conducting TFU on the comparison unit, leading to some patients being called more than 3 times. This study did not calculate the financial impact of TFU for neurosurgical patients. Furthermore, the sample in this study was obtained from 1 organization and 2 neurosurgical units in a large urban medical center, which limits generalization of results to other hospital settings and patient populations.

\section{Conclusion and Recommendations}

This research study has laid the foundation for additional investigations into the impact of a face-to-face meeting on TFU reach rates, subsequent ED visits, and hospital readmissions for neurosurgical patients. This study increased TFU reach rates and decreased the frequency of phone call attempts by conducting face-to-face meetings with patients before hospital discharge. Although the results were not statistically significant in terms of decreasing subsequent ED visits and reducing hospital readmissions, the outcomes were clinically relevant, with an ultimate decrease in overall health care utilization. Further research is needed to fully understand the different factors associated with successfully reaching patients post hospital discharge. The significant and evolving roles of nurse case managers also warrant further investigation to better understand the impact of registered nurses on reducing health care utilization. There is also a need for discovery of innovative health care modalities that would increase patients' engagement in self-care management at home, which might lead to fewer subsequent
ED visits and hospital readmissions, and result in reductions in health care utilization. Overall, it is essential to understand how to identify patients at highest risk for rehospitalization and to develop methods to engage the hardest to reach patients.

\section{Acknowledgements}

Special thanks are extended to Melissa B. Richardson and the Patient Access Line Department Case Managers of The Johns Hopkins Hospital for supporting this study. The authors also are appreciative to Carrie Price for assisting in the literature search, Kimberly Davis for assisting in the implementation of the project, and Margaret O'Connor for manuscript preparation.

\section{Author Disclosure Statement}

Drs. Vergara, Davis, Sheridan, Sullivan, and Budhathoki declared that there are no conflicts of interest. The project described was supported by grant number 1C1CMS33105301-00 from the US Department of Health and Human Services, Centers for Medicare \& Medicaid Services. The contents of this publication are solely the responsibility of the authors and do not necessarily represent the official views of the US Department of Health and Human Services or any of its agencies. The research presented was conducted by the awardee. Results may or may not be consistent with or confirmed by the findings of the independent evaluation contractor.

\section{References}

1. Centers for Medicare and Medicaid. Health Care Innovation Awards: Maryland. 2019. https://innovation.cms.gov/ initiatives/Health-Care-Innovation-Awards/Maryland.html Accessed February 18, 2019. 
2. Berkowitz SA, Parashuram S, Rowan K, et al. Association of a care coordination model with health care costs and utilization: the Johns Hopkins Community Health Partnership (J-CHiP). JAMA Netw Open 2018;1:e184273.

3. Berkowitz SA, Brown P, Brotman DJ, et al. Case study: Johns Hopkins Community Health Partnership: a model for transformation. Healthc (Amst) 2016;4:264-270.

4. Hsiao YL, Bass EB, Wu AW, et al. Implementation of a comprehensive program to improve coordination of care in an urban academic health care system. J Health Organ Manag 2018;32:638-657.

5. Brittain S, Dennis S, Gill A, et al. Patient Access Line. www.hopkinsmedicine.org/strategic_plan/_docs/poster_ walk_compilation.pdf Accessed February 18, 2019.

6. Vergara FH, Sheridan DJ, Sullivan NJ, Budhathoki C. Improving posthospital discharge telephone reach rates through prehospital discharge face-to-face meetings. Prof Case Manag 2017;22:275-283.

7. Vergara FH, Sullivan NJ, Sheridan DJ, Davis JE. The best practice for increasing telephone outreach: an integrative review. Prof Case Manag 2018;23:307-317.

8. Hoyer EH, Brotman DJ, Apfel A, et al. Improving outcomes after hospitalization: a prospective observational multicenter evaluation of care coordination strategies for reducing 30-day readmissions to Maryland hospitals. J Gen Intern Med 2018;33:621-627.

9. Gray JR, Grove SK, Sutherland S. Burns and Grove's: the practice of nursing research, 8th edition. St. Louis, MO: Elsevier, 2017.

10. Meleis AI, Sawyer LM, Im EO, Hilfinger-Messias DK, Schumacher K. Experiencing transitions: an emerging middle-range theory. Adv Nurs Sci 2000;23:12-28.

11. Kuriyama A, Takahashi N, Nakayama T. Reporting of critical care trial abstracts: a comparison before and after the announcement of CONSORT guideline for abstracts. Trials 2017;18:32.

12. IBM SPSS statistics for Windows [Computer software]. Version 25.0. Armonk, NY: IBM Corp., 2017.

13. Cohen J. Statistical power analysis for the behavioral sciences. Hillsdale, NJ: Lawrence Erlbaum Associates, 1988.

14. Pett MA. Nonparametric statistics for health care research: statistics for small samples and unusual distributions, 2nd edition. Thousand Oaks, CA: Sage, 2016.

15. Mosa AS, Yoo I, Parker JC. Online electronic data capture and research data repository system for clinical and translational research. MO Med 2015;112:46-52.

16. Johns Hopkins Medicine Institutional Review Board. HIPPA questions and answers relating to research. 2015. https://www .hopkinsmedicine.org/institutional_review_board/hipaa_ research/faq_research.html Accessed February 21, 2019.

17. Coleman EA, Parry C, Chalmers S, Min SJ. The care transitions intervention: results of randomized controlled trial. Arch Intern Med 2006;166:1822-1828.

18. Parry C, Min SJ, Chugh A, Chalmers S, Coleman EA. Further application of the care transitions intervention: results of randomized controlled trial conducted in a fee-forservice setting. Home Health Care Serv Q 2009;28:84-99.

19. Kind AJ, Jensen L, Barczi S, et al. Low-cost transitional care with nurse managers making mostly phone contact with patients cut rehospitalization at a VA hospital. Health Aff (Millwood) 2012;31:2659-2668.
20. Kind AJ, Brenny-Fitzpatrick M, Leahy-Gross K, et al. Harnessing protocolized adaptation in dissemination: successful implementation and sustainment of the Veterans Affairs Coordinated-Transitional Care Program in a nonVeterans Affairs hospital. J Am Geriatr Soc 2016;64:409-416.

21. Rodak S. Hospitalists, call center play crucial role in reducing readmissions. 2012. www.beckershospitalreview .com/quality/hospitalists-call-center-play-crucial-role-inreducing-readmissions.html Accessed February 18, 2019.

22. Menchine M, Oberfoell S, Schriger D, Walker C, Riddell J, Arora S. Improving telephone follow-up for patients discharged from the emergency department: results of a randomized controlled trial. Acad Emerg Med 2013;20:456-462.

23. Cusimano MD, Pshonyak I, Lee MY, Ilie G. A systematic review of 30-day readmission after cranial neurosurgery. J Neurosurg 2016;127:342-352.

24. Moghavem N, Morrison D, Ratliff JK, HernandezBoussard T. Cranial neurosurgical 30-day readmissions by clinical indication. J Neurosurg 2015;123:189-197.

25. Marcus LP, McCutcheon BA, Noorbakhsh A, et al. Incidence and predictors of 30-day readmission for patients discharged home after craniotomy for malignant supratentorial tumors in California (1995-2010). J Neurosurg 2014;120:1201-1211.

26. Nuño M, Ly D, Mukherjee D, Ortega A, Black KL, Patil CG. Quality of surgical care and readmission in elderly glioblastoma patients. Neurooncol Pract 2014;1:33-39.

27. Bernatz JT, Anderson PA. Thirty-day readmission rates in spine surgery: systematic review and meta-analysis. Neurosurg Focus 2015;39:E7.

28. Webb ML, Nelson SJ, Save A, et al. Of 20,376 lumbar discectomies, $2.6 \%$ of patients readmitted within 30 days: surgical site infection, pain, and thromboembolic events are the most common reasons for readmission. Spine (Phila $\mathrm{Pa}$ 1976) 2016;42:1267-1273.

29. Puvanesarajah V, Nourbakhsh A, Hassanzadeh H, Shimer AL, Shen FH, Singla A. Readmission rates, reasons, and risk factors in elderly patients treated with lumbar fusion for degenerative pathology. Spine (Phila Pa 1976) 2016;41: 1933-1938.

30. Mabud T, Norden J, Veeravagu A, et al. Complications, readmissions, and revisions for spine procedures performed by orthopedic surgeons versus neurosurgeons: a retrospective, longitudinal study. Clin Spine Surg 2016;30:E1376-E1381.

31. Anderson J. Nurse interventions marks 5 years of smoothing transitions of care. Hopkins Insider 2018:1-2. https://www .hopkinsmedicine.org/news/publications/_docs/Hopkins\%20 Insider_September\%202018_FINAL.pdf Accessed April 29, 2019.

32. Holland DE, Brandt C, Targonski PV, Bowles KH. Validating performance of a hospital discharge planning decision tool in community hospitals. Prof Case Manag 2017; 22:204-213.

Address correspondence to: Franz H. Vergara, PhD, DNP, RN, ONC, CCM Bloomberg School of Public Health The Johns Hopkins University 615 North Wolfe Street Baltimore, MD 21205

E-mail: fvergar1@jhmi.edu 